\title{
Tocilizumab: A promising treatment for COVID-19 patients
}

Janani Anandan ${ }^{1}$ and Cecilia Lee $^{2}$

10.17975/sfi-2021-010

\author{
${ }^{1}$ The Woodlands Secondary School, Ontario, Canada \\ 2 Department of Psychology, University of British Columbia, British Columbia, Canada \\ KEYWORDS: COVID-19, tocilizumab, cytokine, interleukin-6
}

\section{INTRODUCTION}

A novel Coronavirus was identified in China as SARSCoV-2 in December 2019 and was later declared a pandemic by the World Health Organization [1]. In most patients, COVID-19 presents with mild or moderate symptoms such as fever, cough, fatigue, and myalgia. However, around $10 \%$ of patients develop more severe symptoms such as pneumonia and multiorgan failure, which can result in death [1-2]. Until vaccines are widely available, the only way to reduce fatality rates is to treat affected patients with existing drugs and therapy options.

Most COVID-19 related deaths are due to an immune overreaction within the body, known as a cytokine storm [3]. Cytokines are proteins that aid in immune responses and are responsible for triggering symptoms such as fever, inflammation, and bodily aches [4]. In a cytokine storm, proteins such as the proinflammatory interleukin-6 (IL-6) are overproduced, which are key in triggering acute inflammation of multiple organs leading to death. Depending upon the condition of the patient, a wide variety of treatment methods are being studied, including remdesivir, lopinavir/ritonavir, interferon beta and other combination therapies [5]. Some of these drugs are used for mild to moderate presentations of the disease, whereas others are administered for patients in adverse conditions with respiratory rates $\geq 30$ breaths $/ \mathrm{min}$ or oxygen saturation $\leq 93 \%$. For severe COVID-19 patients, one such treatment option is tocilizumab; a humanized monoclonal antibody [6]. In the past, monoclonal antibody agents have also been studied as treatments for MERS-CoV with exceptionally potent neutralizing activity [7].

Tocilizumab, a drug approved for cytokine release syndrome, is often used for the treatment of arthritis [1]. Numerous studies have observed evidence that tocilizumab can reduce overall mortality rates in COVID-19 patients [1, 8-12]. As shown in Figure 1, it inhibits the IL-6 receptor, which makes it a promising treatment to control cytokine storms.

\section{RESULTS OF CLINICAL STUDIES}

\section{Mortality rate}

Tocilizumab clinical trials conducted in France, Italy, and the United States (US) for severe COVID-19 patients recorded a significant reduction in mortality rates compared to standard therapeutics [8-10]. In France, a retrospective casecontrolled study with 45 COVID-19 patients was done. The outcome of both tocilizumab patients and the standard care group were compared using both mortality and ICU admission rates [8]. A standard care group of 25 patients received antiviral drugs such as lopinavir-ritonavir, anti-rheumatic drugs like hydroxychloroquine and antibiotics. Whereas the experimental group of 20 patients received one or two doses of tocilizumab in addition to standard care. Notably, the tocilizumab group had higher levels of comorbidities, with a mean Charlson Comorbidity Index (the sum of weights of comorbid diseases other than COVID-19) of 5.3, compared to the standard care group's index of 3.4. In addition to that, $75 \%$ of patients in the tocilizumab group were above 70 years of age compared to $44 \%$ in the standard group. Despite the greater risk factors in the tocilizumab group, the study observed a marked difference in both mortality and ICU admission rates between the groups. Only $25 \%$ of patients in the tocilizumab group died, compared to the $48 \%$ in the standard group. Significantly, $0 \%$ of tocilizumab-treated patients were admitted to the ICU, as compared to $44 \%$ of patients receiving standard care $(p<0.001)$ [8].

Trials conducted in Italy included a cohort study with two groups: a control group of 23 patients and a tocilizumab experimental group of 62 patients. Patients in both the control and experimental groups were given hydroxychloroquine, lopinavir and ritonavir as a standard care treatment [9]. About $57 \%$ of the tocilizumab group were given doses of tocilizumab intravenously, and the rest of them received it subcutaneously. Comparing both groups, 11 of the 23 control patients died, whereas only 2 of the 62 tocilizumab patients 


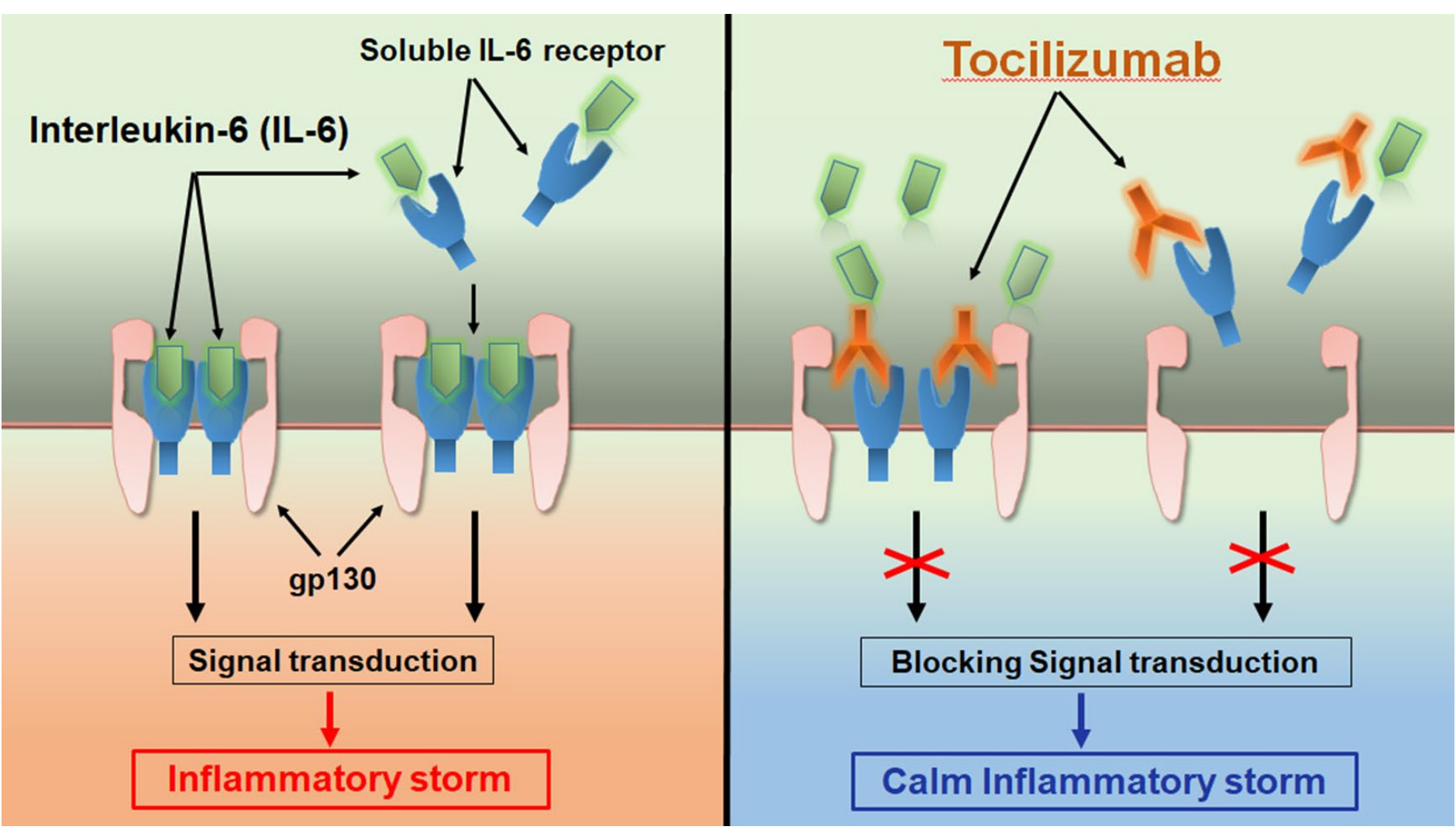

Figure 1. Tocilizumab inhibits IL-6 receptors, controlling cytokine storms. Original figure from Fu et al. [2] in Springer Nature and reproduced under CC BY 4.0 license.

died. Additionally, $92 \%$ of patients in the tocilizumab group were discharged after full recovery. However, only $42 \%$ in the control group recovered. The study noted that the treatment effects were independent of age, sex, and comorbidities such as diabetes, hypertension, and heart disease, and it was concluded that administration of tocilizumab reduced mortality rates drastically $(p=0.004)$ [9].

Furthermore, a study based in the US tested tocilizumab on 153 individuals. In addition to tocilizumab, they were given hydroxychloroquine and glucocorticoids. The 14-day survival rate of patients who were given tocilizumab was $91 \%$ in nonsevere COVID-19 cases and $83 \%$ in severe cases (severe is defined by $\geq 3 \mathrm{~L}$ of supplemental oxygen required for oxygen saturation to be maintained above $93 \%$ ). However, it was noted that this difference was not statistically significant ( $p$ $=0.11$ ) [10], and the mortality rates for both non-severe (9\%) and severe cases (17\%) were lower than that of hospitalized COVID-19 patients in the US (22\%) [11]. This suggests that tocilizumab may be effective for both patients with nonsevere and severe conditions of COVID-19.

Several other global clinical trials were done to study the effectiveness of tocilizumab (Table 1) [1, 12]. During a trial conducted in Qatar, tocilizumab was administered to patients only when they were in the ICU. $12 \%$ of them died, which is markedly lower than the average death rate for COVID-19 patients admitted to the ICU elsewhere (26-50\% as per other studies) [1]. In a third cohort study conducted with 544 patients in Italy, $20 \%$ of the standard care patients died. However, only $7 \%$ of intravenously and $8 \%$ of subcutaneously administered tocilizumab patients died [12]. In both studies, the mortality rate for the tocilizumab group was lower than that of the control/standard treatment group for COVID-19 $[1,12]$.

\section{CRP serum levels}

C-reactive proteins (CRPs) are produced by the liver and released into the bloodstream in response to inflammation, thereby acting as non-specific inflammatory markers. As mentioned earlier, inflammation is triggered during a cytokine storm. As a result, serum CRP levels can increase up to hundred or even thousand-fold in some cases [13]. Since tocilizumab plays a crucial role in the inhibition of the 
Table 1. Comparison of mortality rates in clinical trials studied investigating tocilizumab as the treatment for COVID-19 in humans.

\begin{tabular}{lcccc}
\hline \multicolumn{1}{c}{ Location } & $\begin{array}{c}\text { Patients in } \\
\text { Standard Care } \\
\text { Group (n) }\end{array}$ & $\begin{array}{c}\text { Patients in } \\
\text { Tocilizumab } \\
\text { Group (n) }\end{array}$ & $\begin{array}{c}\text { Mortality Rate } \\
\text { in Standard Care } \\
\text { Group (\%) }\end{array}$ & Mortality Rate in Tocilizumab Group (\%) \\
\hline France & 25 & 20 & 48 & 25 \\
Italy (Clinical Trial 1) & 23 & 62 & 48 & 3 \\
Italy (Clinical Trial 2) & 365 & 179 & 20 & $\begin{array}{c}\text { intravenously administered }=7 \\
\text { subcutaneously administered }=8\end{array}$ \\
Qatar & N/A & 25 & $26-50^{\text {a }}$ & 12 \\
United States & N/A & 153 & $22^{\text {b }}$ & $\begin{array}{c}\text { non-severe }=9 \\
\text { severe }=17\end{array}$ \\
\hline
\end{tabular}

a COVID-19 ICU mortality rate elsewhere

${ }^{\mathrm{b}}$ Hospitalized COVID-19 patients in the US

proinflammatory cytokine IL-6, CRP levels should decrease after the patient is administered this drug. A clinical trial conducted by $\mathrm{Xu}$ et al. resulted in $84 \%$ of patients experiencing a significant decrease in CRP levels, indicating reduced inflammation [14]. In the other trials, the median CRP level decreased from 126.9-193.0 mg/L to 7.9-11.2 mg/L after the administration of tocilizumab, indicating reduced disease severity $[1,15]$.

\section{Mechanical ventilation}

Among other outcomes, tocilizumab administration also observes overall decreases in the number of patients needing mechanical ventilation. In an Italian observational study, out of the 100 patients requiring ventilatory support, 77 experienced a reduced need for oxygen supplementation or no oxygen therapy [6]. Apart from this, in a study conducted in the US on 27 patients ( 21 of which required mechanical ventilation), 15 were extubated after a median of 8 days post-tocilizumab administration [13]. Additionally, in Qatar, $84 \%$ of patients required invasive ventilation on day one, which decreased to only $28 \%$ on day 14 [2]. Overall, in many trials conducted, a remarkable number of patients no longer needed mechanical ventilation at the end of the trial period $[1,12,14]$.

\section{Side effects}

While tocilizumab was highly effective in treating COVID-19, clinical studies also noted a high rate of hospitalacquired infections in patients who were given this drug compared to standard care groups [1, 10, 12]. Although most of these infections were not severe, a detailed analysis of the potential risks and side effects associated with the administration of tocilizumab as a therapy for COVID-19 is still pending. With only less than $1 \%$ of infections being severe (due to liver failure from herpes simplex virus), the benefits of using tocilizumab seem to outweigh the risks for patients hospitalized with COVID-19 until an alternative therapy or vaccination is in place [12].

\section{CONCLUSION}

In all the trials mentioned, tocilizumab was administered in addition to the standard treatments approved. Most standard treatments involved a combination of antiviral drugs (e.g., remdesivir, lopinavir, ritonavir), anti-inflammatory drugs (e.g., glucocorticoids), antibiotics and anti-rheumatic drugs (such as hydroxychloroquine) $[12,14]$. Globally, tocilizumab use showed clinical benefits such as reduced mortality rates and reduced risk of mechanical or invasive ventilation irrespective of the type of standard care treatment given. As all these clinical trials involved under 600 patients, larger studies will be necessary to validate the outcomes. The dosage in each trial varied widely due to supply limitations. Some trials used both intravenous and subcutaneous administration and found that the method of administration did not affect the outcome [12]. In addition, the time of administration of the drug and the selection criteria of the patients differed in each study. Despite these differences, many studies observed a decrease in the mortality rate and invasive ventilation risk $[1,6,12-13]$. Drastic reduction in serum CRP levels indicating decreasing inflammation was also observed [1, 14-15]. Even with consideration of potential side effects, tocilizumab has demonstrated high promise in treating COVID-19 and should 
be considered for inclusion to standard care treatments globally.

\section{REFERENCES}

1. Alattar R, Ibrahim TBH, Shaar SH, Abdalla S, Shukri K, Daghfal JN, et al. Tocilizumab for the treatment of severe coronavirus disease 2019. Journal of Medical Virology. 2020 May 5;1-8 doi: https://doi.org/10.1002/jmv.25964

2. Fu B, Xu X, Wei H. Why tocilizumab could be an effective treatment for severe COVID-19? Journal of Translational Medicine. 2020 Apr;18(1):1-5. doi: https:// doi.org/10.1186/s12967-020-02339-3

3. Zhang C, Wu Z, Li J-W, Zhao H, Wang G-Q. The cytokine release syndrome in severe COVID-19: interleukin-6 receptor antagonist tocilizumab may be the key to reduce mortality. International Journal of Antimicrobial Agents. 2020 May;55(5):105954. doi: https://doi. org/10.1016/j.ijantimicag.2020.105954

4. Zhang J-M, An J. Cytokines, inflammation, and pain. International Anesthesiology Clinics. 2007 Apr;45(2):2737. doi: 10.1097/AIA.0b013e318034194e

5. Bimonte S, Crispo A, Amore A, Celentano E, Cuomo A, Cascella M. Potential antiviral drugs for SARS-Cov-2 treatment: Preclinical findings and ongoing clinical research. In Vivo. 2020 June; 34:1597-1602. doi: https:// doi.org/10.21873/invivo.11949

6. Crisafulli S, Isgrò V, Corte LL, Atzeni F, Trifirò G. Potential role of anti-interleukin (IL)- 6 drugs in the treatment of COVID-19: Rationale, clinical evidence and risks. BioDrugs. 2020 Jun 15;34:415-422. doi: https://doi. org/10.1007/s40259-020-00430-1

7. Ying T, Li H, Lu L, Dimitrov DS, Jiang S. Development of human neutralizing monoclonal antibodies for prevention and therapy of MERS-CoV infections. Microbes and Infection. 2015 Feb;17(2):142-148. doi: https://doi.org/10.1016/j.micinf.2014.11.008

8. Klopfenstein T, Zayet S, Lohse A, Balblanc J-C, Badie J, Royer $\mathrm{P}-\mathrm{Y}$, et al. Tocilizumab therapy reduced intensive care unit admissions and/or mortality in COVID-19 patients. Médecine Et Maladies Infectieuses. 2020 Aug;50(5):397-400. doi: https://doi.org/10.1016/j. medmal.2020.05.001

9. Capra R, Rossi ND, Mattioli F, Romanelli G, Scarpazza C, Sormani MP, et al. Impact of low dose tocilizumab on mortality rate in patients with COVID-19 related pneumonia. European Journal of Internal Medicine. 2020 Jun;(76):31-35. doi: https://doi.org/10.1016/j. ejim.2020.05.009

10. Price CC, Altice FL, Shyr Y, Koff A, Pischel L, Goshua G, et al. Tocilizumab treatment for cytokine release syndrome in hospitalized patients with coronavirus disease 2019: survival and clinical outcomes. Chest Journal. 2020 Oct 1;158(4):1397-1408. doi: https://doi.org/10.1016/j. chest.2020.06.006

11. Goel S, Jain T, Hooda A, Malhotra R, Johal G, Masoomi R, et al. Clinical characteristics and in-hospital mortality for COVID-19 across the globe. Cardiology and Therapy. 2020 Dec;9(2):553-9. doi: https://doi.org/10.1007/s40119020-00189-0

12. Guaraldi G, Meschiari M, Cozzi-Lepri A, Milic J, Tonelli R, Menozzi $M$, et al. Tocilizumab in patients with severe COVID-19: A retrospective cohort study. The Lancet Rheumatology. 2020 Aug;2(8): 474-484. doi: https://doi. org/10.1016/S2665-9913(20)30173-9

13. Jordan SC, Zakowski P, Tran HP, Smith EA, Gaultier C, Marks G, et al. Compassionate use of tocilizumab for treatment of SARS-CoV-2 pneumonia. Clinical Infectious Diseases. 2020 Dec 15;71(12):3168-73. doi: https://doi. org/10.1093/cid/ciaa812

14. Xu X, Han M, Li T, Sun W, Wang D, Fu B, et al. Effective treatment of severe COVID-19 patients with tocilizumab. Proceedings of the National Academy of Sciences of the United States of America. 2020 May 19; 117(20):1097010975. doi: https://doi.org/10.1073/pnas.2005615117

15. Luo P, Liu Y, Qiu L, Liu X, Liu D, Li J. Tocilizumab treatment in COVID-19: A single center experience. Journal of Medical Virology. 2020 Mar; 92(7):814-818. doi: https://doi.org/10.1002/jmv.25801 